\title{
Mathematical Modeling of Ebola Virus Disease in Bat Population
}

\author{
Zineb EL Rhoubari, ${ }^{1}$ Hajar Besbassi $\mathbb{D}^{\mathbb{D}},{ }^{1}$ Khalid Hattaf $\mathbb{D},{ }^{1,2}$ and Noura Yousfi ${ }^{1}{ }^{1}$ \\ ${ }^{1}$ Laboratory of Analysis, Modeling and Simulation (LAMS), Faculty of Sciences Ben M'sik, Hassan II University, \\ P.O. Box 7955, Sidi Othman, Casablanca, Morocco \\ ${ }^{2}$ Centre Régional des Métiers de l'Education et de la Formation (CRMEF), 20340 Derb Ghalef, Casablanca, Morocco \\ Correspondence should be addressed to Khalid Hattaf; k.hattaf@yahoo.fr
}

Received 4 September 2018; Accepted 14 November 2018; Published 2 December 2018

Academic Editor: J. R. Torregrosa

Copyright (c) 2018 Zineb EL Rhoubari et al. This is an open access article distributed under the Creative Commons Attribution License, which permits unrestricted use, distribution, and reproduction in any medium, provided the original work is properly cited.

\begin{abstract}
Many scientific researches have demonstrated that bats can survive Ebola virus and they represent natural reservoirs of this zoonotic virus. In this study, we develop a generalized epizootic model for the transmission dynamics of Ebola virus disease (EVD) in bat population by taking into account the environment contamination. The transmission process is modeled by two general incidence functions that include many incidence rates used in infectious diseases modeling. We first prove that the model is epidemiologically and mathematically well-posed by showing the existence, positivity, and boundedness of solutions. By analyzing the characteristic equations and constructing suitable Lyapunov functionals, the stability analysis of equilibria is investigated.
\end{abstract}

\section{Introduction}

Ebola virus (EBOV) is a filovirus that belongs to the Filoviridae family and it causes a severe hemorrhagic disease in human and nonhuman primates. EBOV is transmitted to the human population through close contact with the blood, secretions, organs, or other bodily fluids of infected animals such as fruit bats, chimpanzees, gorillas, monkeys, antelopes, and porcupines found ill or dead in the rainforest [1]. The first outbreak of Ebola virus disease (EVD) appeared in 1976 near the Ebola River in the Democratic Republic of Congo formerly Zaire. So far, five species of Ebola have been identified which are Zaire, Sudan, Bundibugyo, Reston, and Taï Forest. The first three species have been associated with large outbreaks in Africa. For example, the virus causing the 2014-2016 West African outbreak belongs to the Zaire ebolavirus species [1]. The EVD dominated international news in 2014 and the World Health Organization (WHO) reported more than 28000 cases worldwide and over 11000 deaths [2].

Bats are recognized as a major reservoir of many viral infections including Ebola virus and other human viral infections, such as measles and mumps [3-5]. These species are long-lived mammals with typically highly synchronous birthing $[6,7]$. Other studies have shown that bats can survive Ebola infection without necessarily becoming ill. For instance, Swanpoel et al. [8] experienced that the Ebola virus replicates in bats without being affected. Also, a seropositive bat for Ebola has demonstrated a long-term survival, living healthy over 13 months after sampling [9]. On the other hand, the mechanism of Ebola transmission to nonhuman primates is still unknown, but Leroy et al. [3] think that it occurs with consumption of contaminated fruits by bodily fluids of infected bats.

Over the past years, several mathematical models have been proposed and developed to describe the dynamics of EVD [10-20]. However, these models have traditionally treated the transmission of the disease in human population and ignored the initial source of Ebola in bats. In addition, they do not take into account the contaminated environment by bodily fluids of infected bats. For these mathematical and biological considerations, we propose a generalized epizootic model for Ebola that is given by the following nonlinear system:

$$
\frac{d S}{d t}=A-\mu S-f(S, I) I-g(S, P) P,
$$




$$
\begin{aligned}
& \frac{d I}{d t}=f(S, I) I+g(S, P) P-(\mu+r) I, \\
& \frac{d R}{d t}=r I-\mu R \\
& \frac{d P}{d t}=\sigma I-\eta P
\end{aligned}
$$

where $S(t), I(t)$, and $R(t)$ represent the numbers of susceptible, infected, and recovered bats at time $t$, respectively. Then the total population of bats is $N(t)=S(t)+I(t)+$ $R(t)$. Further, $P(t)$ represents the concentration of EBOV in the environment at time $t$. The susceptible population increases at recruitment rate $A$ by births or immigration and decreases at the natural mortality rate $\mu$. It also decreases and converts into the infected subpopulation by direct contact with infected bats at rate $f(S, I) I$ or by contact with contaminated environment at rate $g(S, P) P$. Thus, the term $f(S, I) I+g(S, P) P$ is the total infection rate of susceptible population. Moreover, the infected bats recover from Ebola at rate $r$ and die only at the natural mortality rate $\mu$. Finally, the parameter $\sigma$ denotes the deposition rate of EBOV in the environment by infected bats and $\eta$ is the decay rate of EBOV in the environment.

As in [21], we suppose that the general incidence functions $f(S, I)$ and $g(S, P)$ are continuously differentiable in the interior of $\mathbb{R}_{+}^{2}$ and satisfy the following hypotheses:

$$
\begin{gathered}
f(0, I)=0 \\
\frac{\partial f}{\partial S}(S, I)>0, \\
\frac{\partial f}{\partial I}(S, I) \leq 0 \\
g(0, P)=0 \\
\frac{\partial g}{\partial S}(S, P)>0 \\
\frac{\partial g}{\partial P}(S, P) \leq 0
\end{gathered}
$$

for all $S, I \geq 0$.

for all $S, P \geq 0$.

Epidemiologically, the above hypotheses are reasonable and consistent with the reality. In fact, the first assumption $\left(H_{1}\right)$ on the function $f(S, I)$ means that the incidence rate by direct contact with infected bats is equal to zero if there are no susceptible bats. This incidence rate is increasing when the number of infected bats is constant and the number of susceptible bats increases. Also, it is decreasing when the number of susceptible bats is constant and the number of infected bats increases. Similarly, the second assumption $\left(\mathrm{H}_{2}\right)$ on the function $g(S, P)$ means that the incidence rate by contaminated environment is equal to zero if there are no susceptible bats. Besides, this incidence rate is increasing when the concentration of EBOV is constant in the environment and the number of susceptible bats increases. Also, it is decreasing when the number of susceptible is constant and the concentration of EBOV in the environment increases. Therefore, the more susceptible bats are, the more infectious events will occur. However, the higher the number of infected bats or the concentration of EBOV in the environment is, the less infectious events will be. Further, the biological meaning of $\left(H_{1}\right)$ for other diseases is given in $[22,23]$. On the other hand, the schematic representation of model (1) is illustrated in Figure 1.

Since the third equation of system (1) is decoupled from the other equations, it suffices to study the following reduced system:

$$
\begin{aligned}
& \frac{d S}{d t}=A-\mu S-f(S, I) I-g(S, P) P, \\
& \frac{d I}{d t}=f(S, I) I+g(S, P) P-(\mu+r) I, \\
& \frac{d P}{d t}=\sigma I-\eta P,
\end{aligned}
$$

It important to note the simple epizootic model for Ebola proposed by Berge et al. [24] is a special case of (2); it suffices to take $f(S, I)=\beta_{1} S, g(S, I)=\beta_{2} S$, and $r=0$. This simple model used a bilinear incidence rate that is based on the principle of mass action. However, such bilinear incidence rate is not perfect to describe minutely the dynamics of the infectious diseases. Also, Berge et al. [24] assumed that infected bats did not recover during Ebola outbreaks. This last hypothesis has been rejected by many researchers [25-27].

Our main purpose of this study is to study the dynamics of our generalized epizootic model presented by system (2). To do this, we organize the rest of the paper as follows. The next section deals with the well-posedness of the model and the existence of equilibria. The stability analysis of the corresponding equilibria is established in Section 3 by applying direct and indirect Lyapunov method. An application and some numerical simulations are given in Section 4 in order to confirm and illustrate our analytical results. Finally, some biological and mathematical conclusions are summarized in Section 5.

\section{Well-Posedness and Equilibria}

For system (2) to be biologically meaningful, it is necessary to show the nonnegativity and boundedness of solutions.

Theorem 1. The set $\mathbb{R}_{+}^{3}$ is positively invariant with respect to system (2). Furthermore, all solutions of (2) are uniformly bounded in the compact subset

$$
\Gamma=\left\{(S, I, P) \in \mathbb{R}_{+}^{3}: S+I \leq \frac{A}{\mu}, P \leq \frac{\sigma A}{\mu \eta}\right\} .
$$

Proof. We have

$$
\left.\frac{d S}{d t}\right|_{S=0}=A>0,
$$




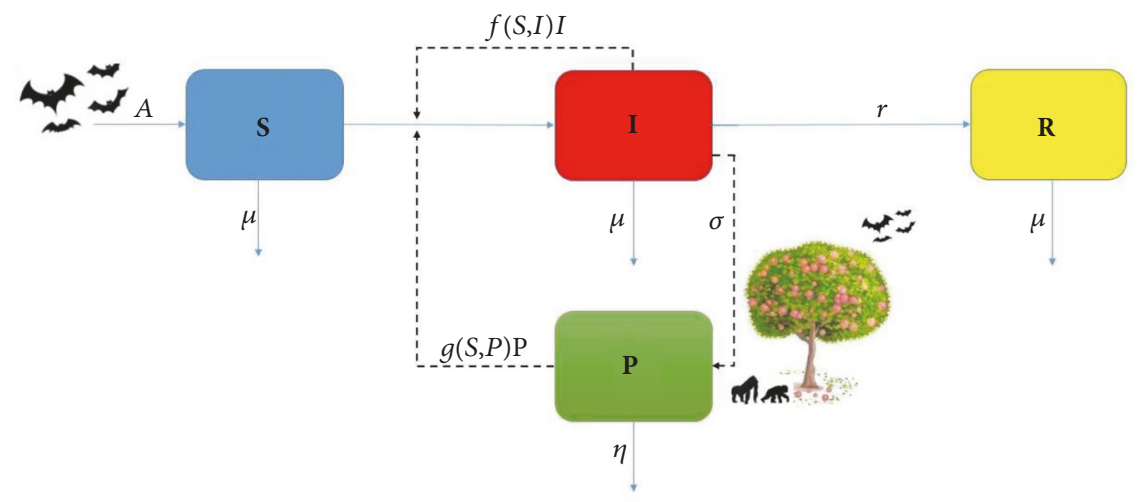

Figure 1: Schematic representation of model (1).

$$
\begin{aligned}
& \left.\frac{d I}{d t}\right|_{I=0}=0, \\
& \left.\frac{d P}{d t}\right|_{P=0}=\sigma I \geq 0, \quad \text { for all } I \geq 0 .
\end{aligned}
$$

This proves the positively invariant property of $\mathbb{R}_{+}^{3}$ with respect to system (2). Let $Q(t)=S(t)+I(t)$, then

$$
\frac{d Q}{d t}=A-\mu S-(\mu+r) I \leq A-\mu Q .
$$

Hence,

$$
\limsup _{t \rightarrow \infty} Q(t) \leq \frac{A}{\mu} .
$$

This implies that $S$ and $I$ are uniformly bounded in the region $\Gamma$. Furthermore, from the bound for $I$ and the last equation of (2), it follows that

$$
\limsup _{t \rightarrow \infty} P(t) \leq \frac{\sigma A}{\mu \eta}
$$

This guarantees the boundedness of $P$. This completes the proof.

Now, we compute the basic reproduction number and prove the existence of two equilibria. Obviously, model (2) always has a disease-free equilibrium $E_{f}(A / \mu, 0,0)$. Then the basic reproduction number of (2) is given by

$$
R_{0}=\frac{\eta f(A / \mu, 0)+\sigma g(A / u, 0)}{\eta(\mu+r)} .
$$

The other equilibrium of system (2) satisfies the following equations:

$$
\begin{array}{r}
A-\mu S-f(S, I) I-g(S, P) P=0, \\
f(S, I) I+g(S, P) P-(\mu+r) I=0, \\
\sigma I-\eta P=0 .
\end{array}
$$

By (9) to (11), we have

$$
f\left(S, \frac{A-\mu S}{\mu+r}\right)+\frac{\sigma}{\eta} g\left(S, \frac{\sigma(A-\mu S)}{\eta(\mu+r)}\right)=\mu+r .
$$

Since $I=(A-\mu S) /(\mu+r) \geq 0$, we have $S \leq A / \mu$. So, there is no equilibrium when $S>A / \mu$. Hence, we define a function $\psi$ on the interval $[0, A / \mu]$ by

$$
\begin{aligned}
\psi(S)= & f\left(S, \frac{A-\mu S}{r+\mu}\right)+\frac{\sigma}{\eta} g\left(S, \frac{\sigma(A-\mu S)}{\eta(r+\mu)}\right)-\mu \\
& -r .
\end{aligned}
$$

We have $\psi(0)=-r-\mu<0, \psi(A / \mu)=(r+\mu)\left(R_{0}-1\right)$, and

$$
\begin{aligned}
\psi^{\prime}(S) & =\frac{\partial f}{\partial S}-\frac{\mu}{r+\mu} \frac{\partial f}{\partial I}+\frac{\sigma}{\eta}\left(\frac{\partial g}{\partial S}-\frac{\mu \sigma}{\eta(r+\mu)} \frac{\partial g}{\partial P}\right) \\
& >0 .
\end{aligned}
$$

Thus, for $R_{0}>1$, there is one endemic equilibrium $E^{*}\left(S^{*}\right.$, $\left.I^{*}, P^{*}\right)$ with $S^{*} \in(0, A / \mu)$ and $I^{*}, P^{*}>0$.

Therefore, we summarize the above discussions in the following result.

Theorem 2. Let $R_{0}$ be defined by (8).

(i) If $R_{0} \leq 1$, then model (2) admits only one disease-free equilibrium $E_{f}\left(S_{0}, 0,0\right)$, where $S_{0}=A / \mu$.

(ii) If $R_{0}>1$, in addition to $E_{f}$, model (2) has a unique endemic equilibrium $E^{*}\left(S^{*}, I^{*}, P^{*}\right)$ with $S^{*} \in(0, A /$ $\mu), I^{*}=\left(A-\mu S^{*}\right) /(\mu+r)$, and $P^{*}=\sigma\left(A-\mu S^{*}\right) / \eta(\mu+$ $r)$.

\section{Stability Analysis}

The aim of this section is to analyze the stability of the two equilibria $E_{f}$ and $E^{*}$. First, we have the following result.

Theorem 3. The disease-free equilibrium $E_{f}$ is globally asymptotically stable when $R_{0} \leq 1$ and it becomes unstable when $R_{0}>1$. 
Proof. To show the first part of this theorem, we consider the following Lyapunov functional:

$$
V(t)=\eta I+g\left(\frac{A}{\mu}, 0\right) P .
$$

Differentiating $V$ with respect to $t$ along the solutions of (2), we get

$$
\begin{aligned}
\left.\dot{V}(t)\right|_{(2)}= & \left(\eta f(S, I)-\eta(\mu+r)+\sigma g\left(\frac{A}{\mu}, 0\right)\right) I \\
& +\eta\left(g(S, P)-g\left(\frac{A}{\mu}, 0\right)\right) P .
\end{aligned}
$$

We have $\lim \sup _{t \rightarrow \infty} S(t) \leq A / \mu$, which implies that each $\omega$-limit point satisfies $S(t) \leq A / \mu$. Hence, it is sufficient to consider solutions for which $S(t) \leq A / \mu$. From (8) and $\left(H_{1}\right)$ $\left(\mathrm{H}_{2}\right)$, we get

$$
\begin{aligned}
\left.\dot{V}(t)\right|_{(2)} & \leq\left(\eta f\left(\frac{A}{\mu}, 0\right)-\eta(\mu+r)+\sigma g\left(\frac{A}{\mu}, 0\right)\right) I \\
& \leq \eta(\mu+r)\left(R_{0}-1\right) I .
\end{aligned}
$$

Consequently, $\left.\dot{V}(t)\right|_{(2)} \leq 0$ for $R_{0} \leq 1$. Furthermore, it is easy to check that the largest invariant subset of $\{(S, I, P) \mid \dot{V}(t)=$ $0\}$ is $\left\{E_{f}\right\}$. By LaSalle's invariance principle [28], we get that $E_{f}$ is globally asymptotically stable when $R_{0} \leq 1$.

In order to show the remaining part, we determine the characteristic equation at the disease-free equilibrium $E_{f}$ that is given by

$$
\begin{aligned}
& (\mu+\lambda)\left(\lambda^{2}+\lambda\left(\eta+\mu+r-f\left(\frac{A}{\mu}, 0\right)\right)\right. \\
& \left.+\eta(\mu+r)\left(1-R_{0}\right)\right)=0 .
\end{aligned}
$$

Let

$$
\begin{aligned}
\Phi(\lambda)= & \lambda^{2}+\lambda\left(\eta+\mu+r-f\left(\frac{A}{\mu}, 0\right)\right) \\
& +\eta(\mu+r)\left(1-R_{0}\right) .
\end{aligned}
$$

We have $\lim _{\lambda \rightarrow+\infty} \Phi(\lambda)=+\infty$ and $\Phi(0)=\eta(\mu+r)\left(1-R_{0}\right)$. If $R_{0}>1$, then $\Phi(0)<0$. Thus, there exists a positive real root of the characteristic equation (18) and hence, the disease-free equilibrium $E_{f}$ is unstable whenever $R_{0}>1$.

For the global stability of the endemic equilibrium $E^{*}$, we assume that $R_{0}>1$ and the functions $f$ and $g$ satisfy, for all $S, I, P>0$, the following hypothesis:

$$
\begin{gathered}
\left(1-\frac{f(S, I)}{f\left(S, I^{*}\right)}\right)\left(\frac{f\left(S, I^{*}\right)}{f(S, I)}-\frac{I}{I^{*}}\right) \leq 0, \\
\left(1-\frac{f\left(S^{*}, I^{*}\right) g(S, P)}{f\left(S, I^{*}\right) g\left(S^{*}, P^{*}\right)}\right) \\
\cdot\left(\frac{f\left(S, I^{*}\right) g\left(S^{*}, P^{*}\right)}{f\left(S^{*}, I^{*}\right) g(S, P)}-\frac{P}{P^{*}}\right) \leq 0 .
\end{gathered}
$$

Theorem 4. If $R_{0}>1$ and $\left(H_{3}\right)$ holds, then the endemic equilibrium $E^{*}$ is globally asymptotically stable.

Proof. Define a Lyapunov functional as follows:

$$
\begin{aligned}
W(t)= & S(t)-S^{*}-\int_{S^{*}}^{S(t)} \frac{f\left(S^{*}, I^{*}\right)}{f\left(X, I^{*}\right)} d X \\
& +I^{*} \Phi\left(\frac{I(t)}{I^{*}}\right)+\frac{g\left(S^{*}, P^{*}\right)}{\eta} P^{*} \Phi\left(\frac{P(t)}{P^{*}}\right) .
\end{aligned}
$$

where $\Phi(X)=X-1-\ln X, X>0$. It is obvious that $\Phi$ attains its strict global minimum at 1 and $\Phi(1)=0$. Then $\Phi(X) \geq 0$ and the functional $W$ is nonnegative.

For convenience, we let $\varphi=\varphi(t)$ for any $\varphi \in\{S, I, P\}$. Differentiating $W$ with respect to $t$ along the solutions of (2), we obtain

$$
\begin{aligned}
\left.\dot{W}(t)\right|_{(2)}= & \left(1-\frac{f\left(S^{*}, I^{*}\right)}{f\left(S, I^{*}\right)}\right) \frac{d S}{d t}+\left(1-\frac{I^{*}}{I}\right) \frac{d I}{d t} \\
& +\frac{g\left(S^{*}, P^{*}\right)}{\eta}\left(1-\frac{P^{*}}{P}\right) \frac{d P}{d t} .
\end{aligned}
$$

Using $A=\mu S^{*}+f\left(S^{*}, I^{*}\right) I^{*}+g\left(S^{*}, P^{*}\right) P^{*}=\mu S^{*}+(\mu+r) I^{*}$ and $\sigma I^{*}=\eta P^{*}$, we get

$$
\begin{gathered}
\left.\dot{W}(t)\right|_{(2)}=\mu S^{*}\left(1-\frac{S}{S^{*}}\right)\left(1-\frac{f\left(S^{*}, I^{*}\right)}{f\left(S, I^{*}\right)}\right) \\
+f\left(S^{*}, I^{*}\right) I^{*}\left(2-\frac{f\left(S^{*}, I^{*}\right)}{f\left(S, I^{*}\right)}-\frac{f(S, I)}{f\left(S^{*}, I^{*}\right)}\right. \\
\left.+\frac{f(S, I) I}{f\left(S, I^{*}\right) I^{*}}-\frac{I}{I^{*}}\right)+g\left(S^{*}, P^{*}\right) P^{*}(3 \\
-\frac{f\left(S^{*}, I^{*}\right)}{f\left(S, I^{*}\right)}+\frac{f\left(S^{*}, I^{*}\right) g(S, P) P}{f\left(S, I^{*}\right) g\left(S^{*}, P^{*}\right) P^{*}}-\frac{P}{P^{*}} \\
\left.-\frac{g(S, P) P I^{*}}{g\left(S^{*}, P^{*}\right) P^{*} I}-\frac{P^{*} I}{P I^{*}}\right) .
\end{gathered}
$$

Thus,

$$
\begin{aligned}
& \left.\dot{W}(t)\right|_{(2)}=\mu S^{*}\left(1-\frac{S}{S^{*}}\right)\left(1-\frac{f\left(S^{*}, I^{*}\right)}{f\left(S, I^{*}\right)}\right) \\
& +f\left(S^{*}, I^{*}\right) I^{*}\left(-1-\frac{I}{I^{*}}+\frac{f\left(S, I^{*}\right)}{f(S, I)}\right. \\
& \left.+\frac{f(S, I) I}{f\left(S, I^{*}\right) I^{*}}\right)+g\left(S^{*}, P^{*}\right) P^{*}\left(-1-\frac{P}{P^{*}}\right. \\
& \left.+\frac{f\left(S, I^{*}\right) g\left(S^{*}, P^{*}\right)}{f\left(S^{*}, I^{*}\right) g(S, P)}+\frac{f\left(S^{*}, I^{*}\right) g(S, P) P}{f\left(S, I^{*}\right) g\left(S^{*}, P^{*}\right) P^{*}}\right) \\
& -f\left(S^{*}, I^{*}\right) I^{*}\left[\Phi\left(\frac{f\left(S^{*}, I^{*}\right)}{f\left(S, I^{*}\right)}\right)\right. \\
& \left.+\Phi\left(\frac{f(S, I)}{f\left(S^{*}, I^{*}\right)}\right)+\Phi\left(\frac{f\left(S, I^{*}\right)}{f(S, I)}\right)\right]-g\left(S^{*}, P^{*}\right)
\end{aligned}
$$




$$
\begin{aligned}
& \cdot P^{*}\left[\Phi\left(\frac{f\left(S^{*}, I^{*}\right)}{f\left(S, I^{*}\right)}\right)+\Phi\left(\frac{P^{*} I}{P I^{*}}\right)\right. \\
& +\Phi\left(\frac{g(S, P) P I^{*}}{g\left(S^{*}, P^{*}\right) P^{*} I}\right) \\
& \left.+\Phi\left(\frac{f\left(S, I^{*}\right) g\left(S^{*}, P^{*}\right)}{f\left(S^{*}, I^{*}\right) g(S, P)}\right)\right]
\end{aligned}
$$

By using $\left(H_{1}\right)$, we easily obtain the following inequality:

$$
\left(1-\frac{S}{S^{*}}\right)\left(1-\frac{f\left(S^{*}, I^{*}\right)}{f\left(S, I^{*}\right)}\right) \leq 0 .
$$

From $\left(H_{3}\right)$, we obtain

$$
\begin{aligned}
-1 & -\frac{I}{I^{*}}+\frac{f\left(S, I^{*}\right)}{f(S, I)}+\frac{f(S, I) I}{f\left(S, I^{*}\right) I^{*}} \\
& =\left(1-\frac{f(S, I)}{f\left(S, I^{*}\right)}\right)\left(\frac{f\left(S, I^{*}\right)}{f(S, I)}-\frac{I}{I^{*}}\right) \leq 0
\end{aligned}
$$

and

$$
\begin{aligned}
-1 & -\frac{P}{P^{*}}+\frac{f\left(S, I^{*}\right) g\left(S^{*}, P^{*}\right)}{f\left(S^{*}, I^{*}\right) g(S, P)} \\
& +\frac{f\left(S^{*}, I^{*}\right) g(S, P) P}{f\left(S, I^{*}\right) g\left(S^{*}, P^{*}\right) P^{*}} \\
& =\left(1-\frac{f\left(S^{*}, I^{*}\right) g(S, P)}{f\left(S, I^{*}\right) g\left(S^{*}, P^{*}\right)}\right) \\
& \cdot\left(\frac{f\left(S, I^{*}\right) g\left(S^{*}, P^{*}\right)}{f\left(S^{*}, I^{*}\right) g(S, P)}-\frac{P}{P^{*}}\right) \leq 0 .
\end{aligned}
$$

Since $\Phi(X) \geq 0$ for $X>0$, we deduce that $\left.\dot{W}\right|_{(2)} \leq 0$ and the equality occurs at $E^{*}$. Consequently, the global asymptotic stability of $E^{*}$ follows from LaSalle's invariance principle.

\section{Application}

The main purpose of this section is to apply our previous results to the following model:

$$
\begin{aligned}
& \frac{d S}{d t}=A-\mu S-\frac{\beta_{1} S I}{1+\alpha_{1} I}-\frac{\beta_{2} S P}{1+\alpha_{2} P}, \\
& \frac{d I}{d t}=\frac{\beta_{1} S I}{1+\alpha_{1} I}+\frac{\beta_{2} S P}{1+\alpha_{2} P}-(\mu+r) I, \\
& \frac{d P}{d t}=\sigma I-\eta P,
\end{aligned}
$$

where $\beta_{1}$ and $\beta_{2}$ are the infection rates caused by infected bats and contaminated environment, respectively. The nonnegative constants $\alpha_{1}$ and $\alpha_{2}$ measure the saturation effect. The remaining parameters have the same biological significance as those in system (2).
Clearly, system (27) is a particular case of (2) with $f(S, I)=\beta_{1} S /\left(1+\alpha_{1} I\right)$ and $g(S, P)=\beta_{2} S /\left(1+\alpha_{2} P\right)$. Based on the previous sections, system (27) has one disease-free equilibrium $E_{f}(A / \mu, 0,0)$, and a unique endemic equilibrium $E^{*}\left(S^{*}, I^{*}, P^{*}\right)$ when $R_{0}=A \beta_{1} / \mu(\mu+r)+\sigma A \beta_{2} / \mu \eta(\mu+$ $r)>1$. In addition, it is clear that the hypotheses $\left(H_{1}\right)-\left(H_{3}\right)$ are checked for both incidence functions $f$ and $g$. Applying Theorems 3 and 4, we deduce the following corollary.

\section{Corollary 5.}

(i) When $R_{0} \leq 1$, the disease-free equilibrium $E_{f}$ of model (27) is globally asymptotically stable.

(ii) When $R_{0}>1, E_{f}$ becomes unstable and the endemic equilibrium $E^{*}$ of model (27) is globally asymptotically stable.

Now, we simulate system (27) with the following parameter values: $A=1.5, \mu=0.0003, \beta_{1}=4.5 \times 10^{-6}, \beta_{2}=$ $1.3 \times 10^{-4}, \alpha_{1}=0.01, \alpha_{2}=0.01, r=0.041, \sigma=0.02$, and $\eta=0.8$. By a simple calculation, we get $R_{0}=0.9383$. Then system (27) has one disease-free equilibrium $E_{f}(5000,0,0)$. It follows from Corollary 5(i) that $E_{f}$ is globally asymptotically stable and the solution of (27) converges to $E_{f}$ as shown in Figure 2. In this case, the disease dies out.

Next, we simulate the case when the basic reproduction number is bigger than one. For this end, we choose $\beta_{1}=$ $2.5 \times 10^{-5}$ and do not change the other parameter values. We get $R_{0}=3.4201>1$. Hence, system (27) has a unique endemic equilibrium $E^{*}(1759.8457,23.5361,0.5887)$. From (ii) of Corollary $5, E^{*}$ is globally asymptotically stable, which means that the Ebola virus persists in the bat population and the disease becomes endemic. This result is illustrated by Figure 3.

\section{Conclusions}

In this article, we have proposed and investigated a new generalized epizootic model that describes the transmission dynamics of Ebola virus disease in bat population. The transmission process from bat-to-bat and contaminated environment-to-bat is modeled by two general nonlinear functions which cover many special cases using in the previous studies such as the saturated incidence, the classical bilinear incidence, the Beddington-DeAngelis functional response, the Hattaf-Yousfi functional response, and the Crowley-Martin functional response. The well-posedness of the proposed model and the stability analysis of equilibria are rigorously studied. More precisely, we have established the existence, uniqueness, nonnegativity, and boundedness of solutions. By using appropriate Lyapunov functionals and linearization technique, we have proved the first steady state $E_{f}$ is globally asymptotically stable when $R_{0} \leq 1$, which means that the disease dies out in the bat population. However, when $R_{0}>1, E_{f}$ becomes unstable and the model has an endemic steady state $E^{*}$ which is globally asymptotically stable. This leads to the persistence of disease in the bat population when $R_{0}>1$. 

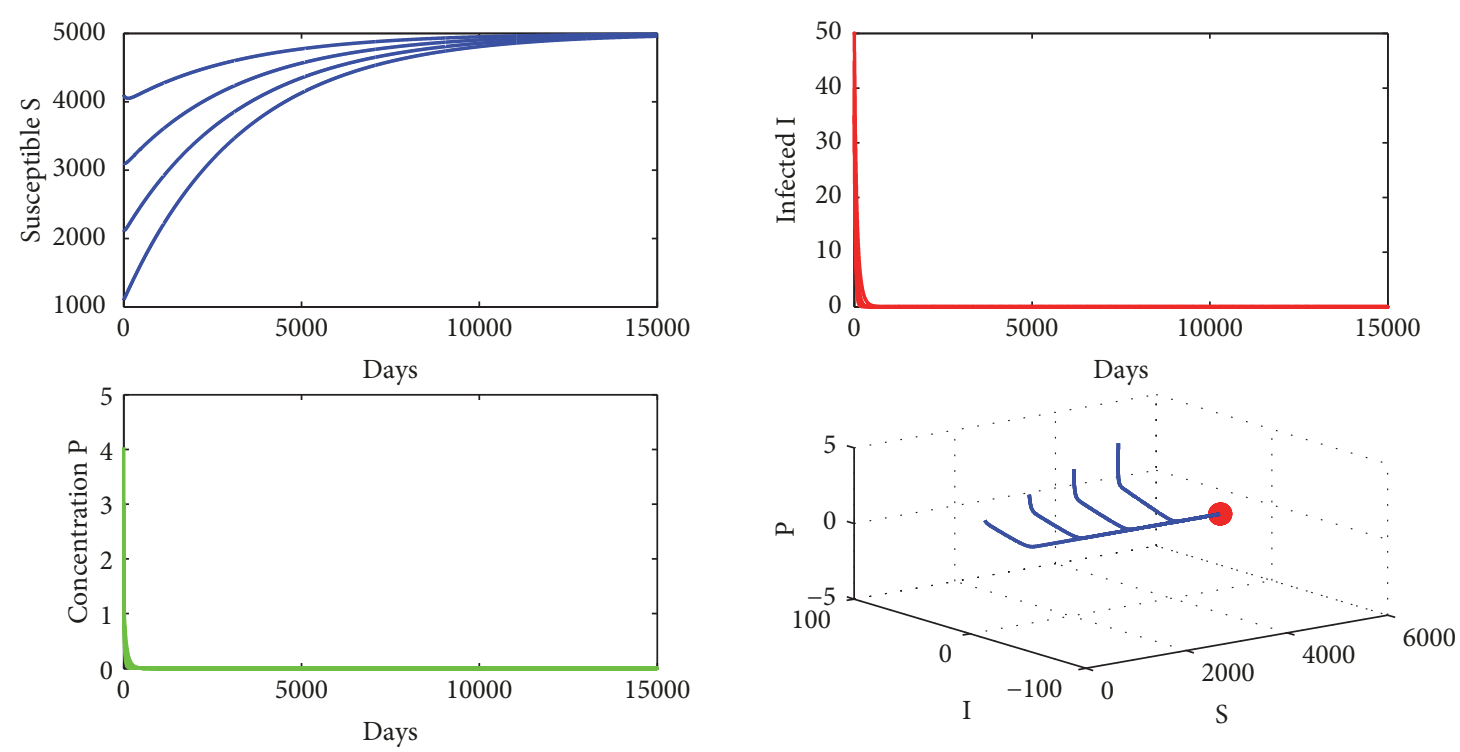

FIgURE 2: Demonstration of the global stability of the disease-free equilibrium $E_{f}$ for $R_{0}=0.9383 \leq 1$.
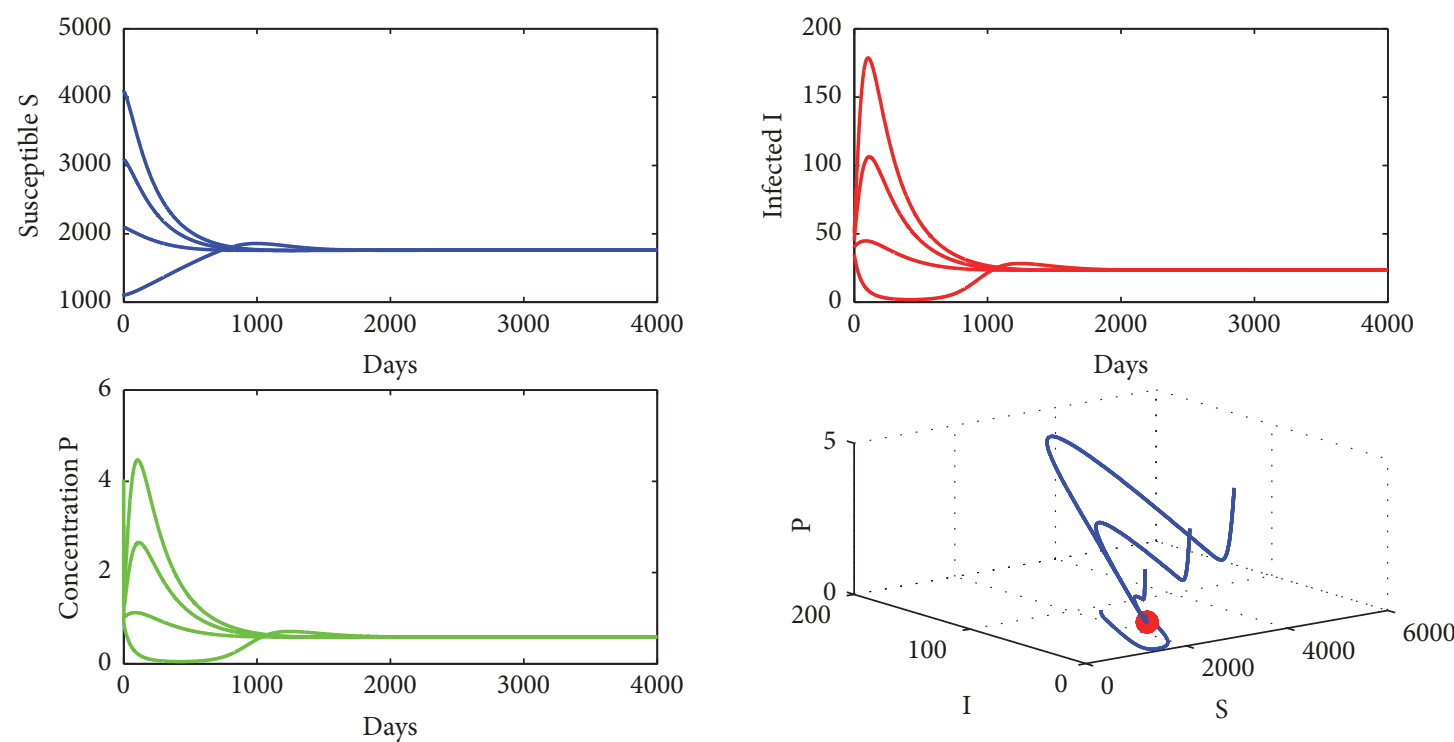

Figure 3: Demonstration of the global stability of the endemic equilibrium $E^{*}$ for $R_{0}=3.4201>1$.

\section{Data Availability}

The data used to support the findings of this study are available from the corresponding author upon request.

\section{Conflicts of Interest}

The authors declare that they have no conflicts of interest.

\section{References}

[1] WHO, "Ebola virus disease," http://www.who.int/en/newsroom/fact-sheets/detail/ebola-virus-disease.

[2] WHO, "Ebola situation report," http://www.who.int/csr/disease/ebola/situation-reports/archive/en/.
[3] E. M. Leroy, B. Kumulungui, X. Pourrut et al., "Fruit bats as reservoirs of Ebola virus," Nature, vol. 438, no. 7068, pp. 575576, 2005.

[4] X. Pourrut, M. Souris, J. S. Towner et al., "Large serological survey showing cocirculation of Ebola and Marburg viruses in Gabonese bat populations, and a high seroprevalence of both viruses in Rousettus aegyptiacus," BMC Infectious Diseases, vol. 9, p. 159, 2009.

[5] D. T. S. Hayman, "Bats as Viral Reservoirs," Annual Review of Virology, vol. 3, pp. 77-99, 2016.

[6] D. T. S. Hayman, R. McCrea, O. Restif et al., "Demography of straw-colored fruit bats in Ghana," Journal of Mammalogy, vol. 93, no. 5, pp. 1393-1404, 2012.

[7] E. G. Crichton and P. H. Krutzsch, Krutzsch, Reproductive biology of bats, Academic Press, 2000. 
[8] R. Swanepoel, P. A. Leman, F. J. Burt et al., "Experimental inoculation of plants and animals with Ebola virus," Emerging Infectious Diseases, vol. 2, no. 4, pp. 321-325, 1996.

[9] D. T. S. Hayman, P. Emmerich, M. Yu et al., "Long-term survival of an urban fruit bat seropositive for ebola and lagos bat viruses," PLoS ONE, vol. 5, no. 8, Article ID e11978, 2010.

[10] X.-S. Wang and L. Zhong, "Ebola outbreak in West Africa: realtime estimation and multiple-wave prediction," Mathematical Biosciences and Engineering, vol. 12, no. 5, pp. 1055-1063, 2015.

[11] A. Rachah and D. F. M. Torres, "Mathematical modelling, simulation, and optimal control of the 2014 Ebola outbreak in West Africa," Discrete Dynamics in Nature and Society, vol. 2015, Article ID 842792, 9 pages, 2015.

[12] A. Rachah and D. F. Torres, "Dynamics and optimal control of Ebola transmission," Mathematics in Computer Science, vol. 10, no. 3, pp. 331-342, 2016.

[13] A. Rachah and D. F. Torres, "Predicting and controlling the Ebola infection," Mathematical Methods in the Applied Sciences, vol. 40, no. 17, pp. 6155-6164, 2017.

[14] C. Althaus, "Estimating the reproduction number of Ebola virus (EBOV) during the 2014 outbreak in West Africa," PLOS Currents: Outbreaks, 2014.

[15] G. Chowell, N. W. Hengartner, C. Castillo-Chavez, P. W. Fenimore, and J. M. Hyman, "The basic reproductive number of Ebola and the effects of public health measures: the cases of Congo and Uganda," Journal of Theoretical Biology, vol. 229, no. 1, pp. 119-126, 2004.

[16] E. V. Grigorieva and E. N. Khailov, "Optimal vaccination, treatment, and preventive campaigns in regard to the SIR epidemic model," Mathematical Modelling of Natural Phenomena, vol. 9, no. 4, pp. 105-121, 2014.

[17] I. Area, H. Batarfi, J. Losada, J. J. Nieto, W. Shammakh, and Á. Torres, "On a fractional order Ebola epidemic model," Advances in Difference Equations, vol. 2015, article 278, 2015.

[18] A. K. Jones, "Green computing: new challenges and opportunities," in Proceedings of the on Great Lakes Symposium on VLSI, p. 3, ACM, 2017.

[19] Z. Xia, S. Wang, S. Li et al., "Modeling the transmission dynamics of Ebola virus disease in Liberia," Scientific Reports, vol. 5, no. 1, 2015.

[20] Jia-Ming Zhu, Lu Wang, and Jia-Bao Liu, "Eradication of Ebola Based on Dynamic Programming," Computational and Mathematical Methods in Medicine, vol. 2016, Article ID 1580917, 9 pages, 2016.

[21] K. Hattaf, A. A. Lashari, Y. Louartassi, and N. Yousfi, "A delayed SIR epidemic model with general incidence rate," Electronic Journal of Qualitative Theory of Differential Equations, No. 3, 9 pages, 2013.

[22] X.-Y. Wang, K. Hattaf, H.-F. Huo, and H. Xiang, "Stability analysis of a delayed social epidemics model with general contact rate and its optimal control," Journal of Industrial and Management Optimization, vol. 12, no. 4, pp. 1267-1285, 2016.

[23] K. Hattaf and N. Yousfi, "A numerical method for a delayed viral infection model with general incidence rate," Journal of King Saud University - Science, vol. 28, no. 4, pp. 368-374, 2016.

[24] T. Berge, J. Lubuma, A. J. O. Tassè, and H. M. Tenkam, “Dynamics of host-reservoir transmission of Ebola with spillover potential to humans," Electronic Journal of Qualitative Theory of Differential Equations, vol. 14, pp. 1-32, 2018.

[25] B. R. Amman, S. A. Carroll, Z. D. Reed et al., "Seasonal Pulses of Marburg Virus Circulation in Juvenile Rousettus aegyptiacus Bats Coincide with Periods of Increased Risk of Human Infection," PLoS Pathogens, vol. 8, no. 10, p. e1002877, 2012.

[26] J. Buceta and K. Johnson, "Modeling the Ebola zoonotic dynamics: Interplay between enviroclimatic factors and bat ecology," PLoS ONE, vol. 12, no. 6, 2017.

[27] G. Fiorillo, P. Bocchini, and J. Buceta, "A Predictive Spatial Distribution Framework for Filovirus-Infected Bats," Scientific Reports, vol. 8, no. 1, 2018.

[28] J. P. LaSalle, The Stability of Dynamical Systems, SIAM, Philadelphia, Pa, USA, 1976. 


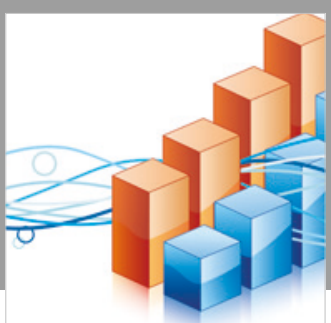

Advances in

Operations Research

\section{-n-m}
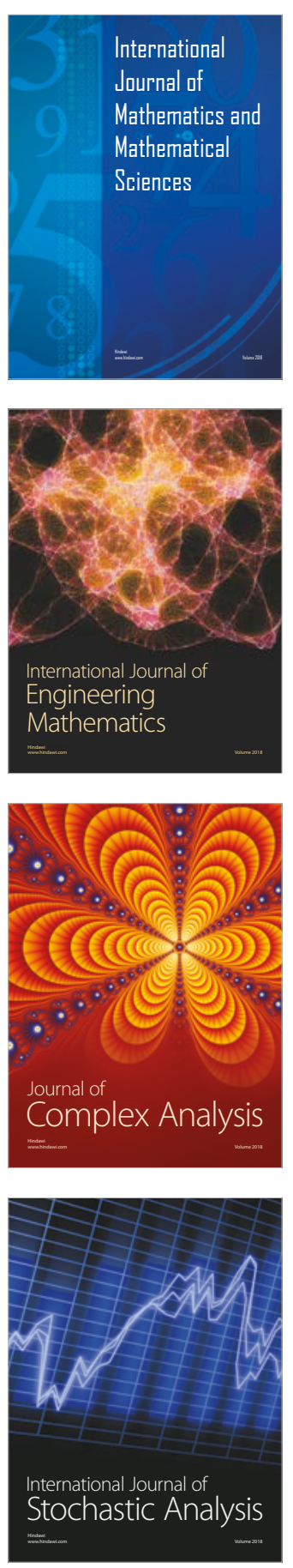
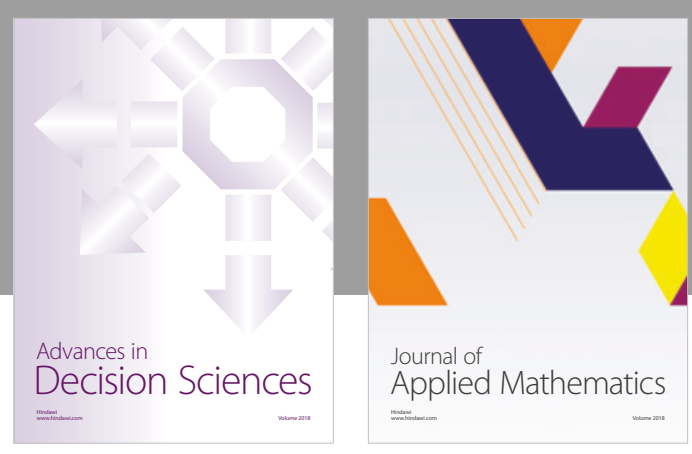

Journal of

Applied Mathematics
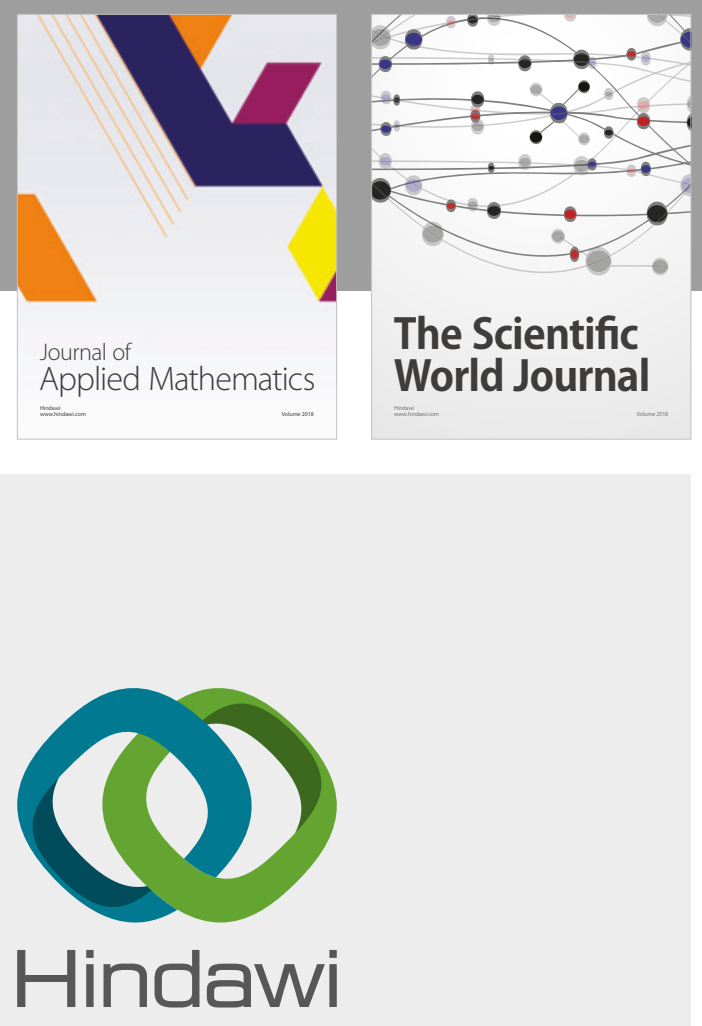

Submit your manuscripts at

www.hindawi.com

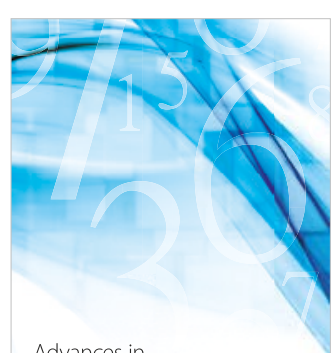

Advances in
Numerical Analysis
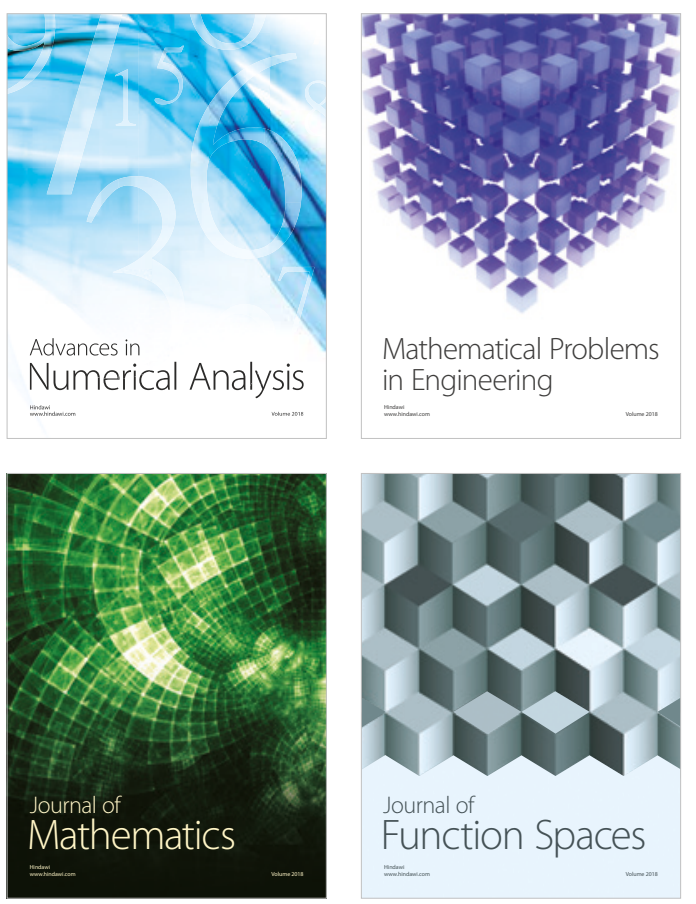

Mathematical Problems in Engineering

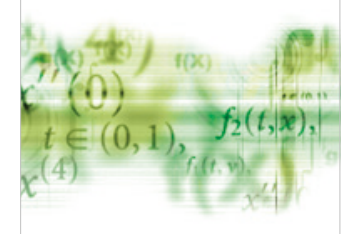

International Journal of

Differential Equations

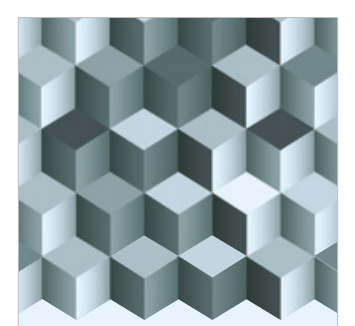

Journal of

Function Spaces

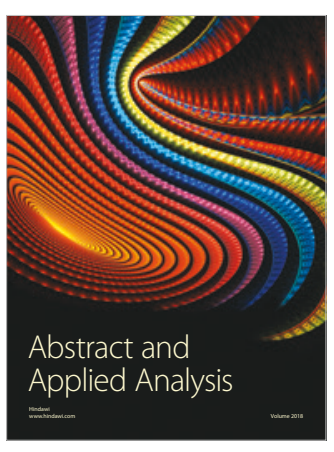

The Scientific

World Journal

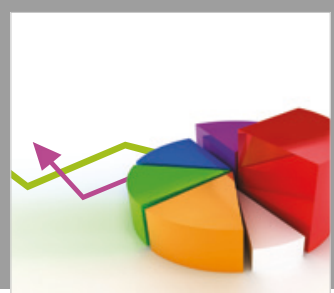

Journal of

Probability and Statistics
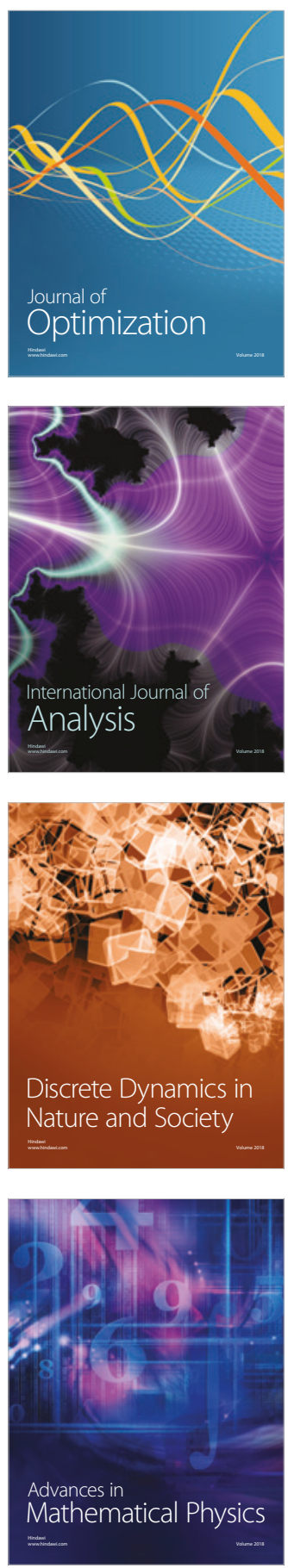